\title{
Doing Justice to Patients with Dementia in ICU Triage
}

Vinay, Rasita ; Baumann, Holger ; Biller-Andorno, Nikola

DOI: https://doi.org/10.1080/15265161.2021.1980147

Posted at the Zurich Open Repository and Archive, University of Zurich ZORA URL: https://doi.org/10.5167/uzh-208378

Journal Article

Published Version

Originally published at:

Vinay, Rasita; Baumann, Holger; Biller-Andorno, Nikola (2021). Doing Justice to Patients with Dementia in ICU Triage. The American Journal of Bioethics, 21(11):71-74.

DOI: https://doi.org/10.1080/15265161.2021.1980147 


\section{Doing Justice to Patients with Dementia in ICU Triage}

\section{Rasita Vinay, Holger Baumann \& Nikola Biller-Andorno}

To cite this article: Rasita Vinay, Holger Baumann \& Nikola Biller-Andorno (2021) Doing Justice to Patients with Dementia in ICU Triage, The American Journal of Bioethics, 21:11, 71-74, DOI: 10.1080/15265161.2021.1980147

To link to this article: https://doi.org/10.1080/15265161.2021.1980147

曲 Published online: 28 Oct 2021.

Submit your article to this journal $\pi$

Q View related articles $₫$

View Crossmark data \lceil 


\section{CONCLUSION}

We welcome and applaud Wilkinson's excellent discussion of the pros and cons of using consideration of frailty in pandemic-driven triage protocols. We object to the very idea of using life-years-saved or longevity as a criterion for rationing, because of its intrinsic relationship to both ageism and ableism. But we accept and welcome the use of frailty diagnoses in rationing, to the extent that such diagnoses are tied to odds of surviving to discharge, or to surviving only for very short periods after discharge. Beyond such short-term considerations, the reversibility of frailty in many cases, and the lack of data about reversibility among the younger frail, militate against the use of frailty as a triage criterion.

\section{DISCLOSURE STATEMENT}

No potential conflict of interest was reported by the author(s).

\section{FUNDING}

The author(s) reported there is no funding associated with the work featured in this article.

\section{REFERENCES}

Chapman, E. N., A. Kaatz, and M. Carnes. 2013. Physicians and implicit bias: how doctors may unwittingly perpetuate health care disparities. Journal of General Internal Medicine 28 (11):1504-10. doi:10.1007/s11606013-2441-1.
Chu, N. M., X. Chen, S. P. Norman, J. Fitzpatrick, S. M. Sozio, B. G. Jaar, A. Frey, M. M. Estrella, Q.-L. Xue, R. S. Parekh, et al. 2020. Frailty prevalence in younger endstage kidney disease patients undergoing dialysis and transplantation. American Journal of Nephrology 51 (7): 501-10. doi:10.1159/000508576.

Collard, R. M., H. Boter, R. A. Schoevers, and R. C. Oude Voshaar. 2012. Prevalence of frailty in community-dwelling older persons: a systematic review. Journal of the American Geriatrics Society 60 (8):1487-92. doi:10.1111/j. 1532-5415.2012.04054.x.

Fried, L. P., C. M. Tangen, J. Walston, A. B. Newman, C. Hirsch, J. Gottdiener, T. Seeman, R. Tracy, W. J. Kop, G. Burke, et al. 2001. Frailty in older adults: Evidence for a phenotype. The Journals of Gerontology. Series A, Biological Sciences and Medical Sciences 56 (3):M146-56. doi:10.1093/gerona/56.3.m146.

Gill, T. M., E. A. Gahbauer, H. G. Allore, and L. Han. 2006. Transitions between Frailty States among communityliving older persons. Archives of Internal Medicine 166 (4):418-23. doi:10.1001/archinte.166.4.418.

Hanlon, P., B. I. Nicholl, B. D. Jani, D. Lee, R. McQueenie, and F. S. Mair. 2018. Frailty and pre-frailty in middleaged and older adults and its association with multimorbidity and mortality: A prospective analysis of 493737 UK Biobank Participants. The Lancet Public Health 3 (7): e323-32. doi:10.1016/S2468-2667(18)30091-4.

Ofori-Asenso, R., K. L. Chin, M. Mazidi, E. Zomer, J. Ilomaki, A. R. Zullo, D. Gasevic, Z. Ademi, M. J. Korhonen, D. LoGiudice, et al. 2019. Global incidence of frailty and prefrailty among community-dwelling older adults: A systematic review and meta-analysis. JAMA Network Open 2 (8):e198398-e98. doi:10.1001/jamanetworkopen.2019.8398.

Wilkinson, Dominic J. C., 2021. Frailty Triage: Is rationing intensive medical treatment on the grounds of frailty ethical? The American Journal of Bioethics 21 (11):48-63. doi:10.1080/15265161.2020.1851809.

\title{
Doing Justice to Patients with Dementia in ICU Triage
}

\author{
Rasita Vinay, Holger Baumann, and Nikola Biller-Andorno \\ University of Zurich
}

In response to the COVID-19 pandemic, countries around the world developed ICU triaging guidelines (Jöbges and Biller-Andorno 2020). One important point of convergence was an explicit commitment to avoid discrimination based on age, disability and social factors (Jöbges et al. 2020). However, what this 
commitment amounts to and what exactly constitutes unjust discrimination in specific cases is a matter of ongoing controversy. In this comment, we will probe the question how we can do justice to patients with dementia in ICU triage.

It is interesting to note how guidelines struggle with this issue. For instance, the first versions of the Swiss guidelines listed severe and moderate dementia within the general exclusion criteria, this was later removed in revisions made in November 2020 to avoid possible constitutional discrimination. At the same time, the Clinical Frailty Scale (CFS) was introduced as a tool for assessing short-term medical prognosis, which then led to concerns about unjust discrimination. It was argued that using the CFS-a clinical tool used to assess the care and support needs of elderly persons-for triaging runs the danger of excluding individuals even with moderate dementia, because of their increased need for everyday support due to their mental condition (Alzheimer Switzerland 2021). ${ }^{1}$ How are we to evaluate such concerns, and how does Wilkinson's paper help us to understand them better?

\section{Frailty and Discrimination}

In the first part of his paper, Wilkinson (2021) elaborates that frailty has been linked to three "potentially highly relevant variables for triage decisions: probability of survival, longevity and quality of life" (55). For this reason, he contends, using the CFS is not (unjustly) discriminatory. Unjust discrimination would only occur if criteria such as age or severe dementia were directly used as exclusion criteria, since they are not linked to survival, longevity, or quality of life in the same way that frailty is. Are allegations about discrimination misguided then if dementia is not directly appealed to?

To establish the claim that using frailty is not unjustly discriminatory, it must first be shown that the three variables which frailty is linked to are not themselves-either on their own or in combinationunjustly discriminatory. While Wilkinson is aware of this point, he does not address it directly and instead refers to the different variables at different points of his discussion. In general, he seems to suggest that it is a potential advantage and part of the justification for using frailty that it can be linked to many variables

\footnotetext{
${ }^{1}$ The Alzheimer's society in UK also published a statement requesting NICE guidelines to avoid using the Clinical Frailty Score for triaging dementia patients, and instead ensuring that clinicians perform a holistic assessment to ensure dementia patients are not disadvantaged in receiving the right care because of their labelled condition (Alzheimer's Society 2021. https://www.alzheimers.org.uk/news/2020-03-30/critical-carepeople-dementia-during-coronavirus-pandemic).
}

(e.g., 11). Such justifications could potentially obscure discussions about discrimination, and we would suggest to first discuss the different variables independently before explicitly examining how they relate to each other. For example, many guidelines used shortterm survival as a primary criterion, and other criteria such as age or quality of life were used as tie-breaker in cases of similar short-term prognosis (Vinay, Baumann, and Biller-Andorno 2021). Wilkinson does not provide such a framework or hierarchy.

Wilkinson also suggests that using "quality of life" on its own is often problematic and can lead to discrimination (56). Like many other bioethicists, we share the view that considerations about quality of life should not be used as a criterion in triage guidelines (although, such considerations may play an important role in a patient's decision toward ICU treatment). Wilkinson is more positive about using longevity or "life-years left" as a criterion, stating that it has intuitive appeal and can draw on considerations about fairness (life-cycle approach), even though some have argued against such approaches. Most of the time, Wilkinson refers to the least controversial criterion in his discussion of discrimination: survival/short-term survival. This variable has been employed as a primary criterion in many triaging guidelines, which brings us to question whether frailty can be justified by appealing to its relation to shortterm survival, and therefore avoids unjust discrimination in the case of dementia patients.

\section{Frailty, Short-Term Survival and Unjust Discrimination}

The first question to be asked is whether using shortterm survival as a criterion for triage constitutes unjust discrimination. We share Wilkinson's view that shortterm medical prognosis as a primary criterion in triage is justified holistically and pragmatically as the criterion that best reconciles different moral values in triage. We also believe that although triage protocols should not further disadvantage vulnerable populations, emergency situations such as a pandemic are not the place to remedy already existing inequalities and injustices (Reid 2020). More specifically, suggestions proposing the prioritization of persons from certain social backgrounds over those from wealthier backgrounds, despite shortterm prognosis, are ethically problematic on grounds of domain-specific fairness and the principle of maximizing benefits. At the same time, it should be emphasized that a pandemic and ICU rationing reminds and requires us to renew our commitment and moral obligation to address social justice issues and to give special attention 
to vulnerable groups. For the context of our discussion, the following relation is important: If frailty is robustly linked to short-term survival, its use in ICU triage does not constitute an unjust discrimination, since shortterm survival cannot be regarded as being unjustly discriminatory.

The second question to be asked is whether frailty (and its clinical assessment) is in fact reliably linked to short-term survival across patient groups. After all, the use of the CFS might work for some groups of patients but not for others. Wilkinson explicitly refers to this possibility, mentioning, e.g., that frailty in younger persons does not reduce the probability of short-term survival in the same way as in old persons, but only in relativity to the group of young persons. ${ }^{2}$ However, Wilkinson does not seem to regard such differences as a general problem for using frailty in ICU triage. By contrast, we argue that this issue and the accompanying danger of (implicit) unjust discrimination needs to be addressed, since it potentially undermines the normative justification and practical relevance of using the CFS in ICU triage.

\section{Doing Justice to Patients with Dementia}

Not using dementia as an explicit exclusion criterion for triage is certainly a step toward avoiding discrimination. However, using a CFS can be seen as a window-dressing if the relationship between frailty and the chosen primary criterion, such as short-term prognosis, is not robust. In this case, it can be questioned if the assumption that the degree of dementia generally corresponds to the degree of frailty, justifies the use of CFS as a triaging tool rather than as a clinical assessment of care needs (Rockwood and Theou 2020). For instance, the current Swiss triaging guidelines use a CFS of 7 and above ("severely frail") in combination with an age over 65 , as well as a CFS of 6 and above ("moderately frail") in combination with an age over 85, as exclusion criterion for ICU admission (Swiss Academy of Medical Sciences 2021). ${ }^{3}$ This means that a 66-year-old person with severe dementia, completely dependent on personal care but physically stable and not at high risk of dying within the next six months, will not be considered for ICU treatment. While there may be good arguments with views to

\footnotetext{
${ }^{2}$ The Clinical Frailty Scale has been developed to summarize the overall level of frailty of older adults.

${ }^{3}$ The guidelines do stress the limited available evidence regarding most appropriate thresholds and the relevance of good clinical judgement in using the scales. This, however, raises question about the practical relevance of the CFS and its advantages over an individualized assessment that uses more fine-grained measures.
}

quality-of-life-related outcomes and the burden of treatment for the patient, it is not obvious how this exclusion criterion relates to short-term prognosis.

Triaging guidelines have the potential to play an important role in safeguarding fair allocation not only in the current pandemic, but also in future public health crises. It is therefore worthwhile to continue refining the guidance that has emerged in the recent past, often in a remarkable effort over a short period of time. A well-argued choice of allocation criteria that are consistently operationalized, and assessment tests or procedures that do not unfairly disadvantage groups such as persons living with dementia, will contribute significantly to emergency preparedness and societal resilience.

\section{DISCLOSURE STATEMENT}

No potential conflict of interest was reported by the author(s).

\section{FUNDING}

RV's $\mathrm{PhD}$ research is funded by the Swiss Government Excellence Scholarship from the Federal Commission for Scholarships for Foreign Students.

\section{REFERENCES}

Alzheimer's Society. 2021. Critical care for people with dementia during the coronavirus pandemic. Accessed August 25, 2021. https://www.alzheimers.org.uk/news/ 2020-03-30/critical-care-people-dementia-during-coronavirus-pandemic

Alzheimer Switzerland. 2021. Aktualisierung der Richtlinien zu Triageentscheidungen bei Überlastung der Intensivstationen: «Mittelschwere» oder «schwere» Demenzerkrankungen sind per se keine Kriterien mehr für die Triage. Accessed August 30, 2021. https://www.alzheimer-schweiz.ch/fileadmin/dam/ Alzheimer_Schweiz/Dokumente/Ueber_Uns/Politik/2021_ 01_21_HaltungALZCH_Triage_DE.pdf.

Jöbges, S., and N. Biller-Andorno. 2020. Ethics guidelines on COVID-19 triage-an emerging international consensus. Critical Care 24 (1):201. doi: 10.1186/s13054-020-02927-1.

Jöbges, S., R. Vinay, V. A. Luyckx, and N. Biller-Andorno. 2020. Recommendations on COVID-19 triage: International comparison and ethical analysis. Bioethics 34 (9):948-59. doi: 10.1111/bioe. 12805.

Reid, L. 2020. Triage of critical care resources in COVID19: A stronger role for justice. Journal of Medical Ethics 46 (8):526-5. doi: 10.1136/medethics-2020-106320.

Rockwood, K., and O. Theou. 2020. Using the clinical frailty scale in allocating scarce health care resources. Canadian Geriatrics Journal 23 (3):210-5. doi: 10.5770/cgj.23.463.

Swiss Academy of Medical Sciences. 2021. Intensive care medicine: Triage under resource scarcity. Accessed August 30, 2021. https://www.samw.ch/en/Ethics/TopicsA-to-Z/Intensive-care-medicine.html. 
Vinay, R., H. Baumann, and N. Biller-Andorno. 2021. Ethics of ICU triage during COVID-19. British Medical Bulletin 138 (1):5-15. doi: 10.1093/bmb/ldab009.
Wilkinson, Dominic J. C., 2021. Frailty Triage: Is Rationing Intensive Medical Treatment on the Grounds of Frailty Ethical? The American Journal of Bioethics 21 (11):48-63. doi:10.1080/15265161.2020.1851809.

\title{
The Complex Relationship between Disability Discrimination and Frailty Scores
}

\author{
Joel Michael Reynolds ${ }^{\mathrm{a}, \mathrm{b}, \mathrm{c}}$ (D), Charles E. Binkley ${ }^{\mathrm{d}}$ (D), and Andrew Shuman ${ }^{\mathrm{e}}$ (ID)

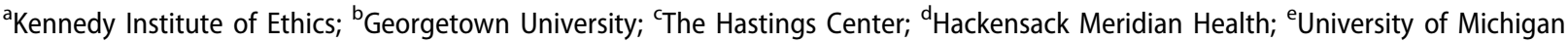 \\ Medical School
}

Wilkinson (2021) argues that the use of frailty scores in ICU triage does not necessarily involve discrimination on the basis of disability. In support of this argument, he claims, "it is not the disability per se that the score is measuring-rather it is the underlying physiological and physical vulnerability" (55). While we appreciate the attention Wilkinson explicitly pays to disability in this piece, we find his use of the distinction between disability and underlying vulnerability untenable both theoretically and practically. We begin with a brief overview of research in philosophy of disability concerning the meaning of the concept itself. We argue that this research demonstrates that many forms of disability do not involve underlying vulnerabilities, and, furthermore, that Wilkinson equivocates between "disability" understood as a medical category vs. "disability" understood as a feature of lived experience. We reject Wilkinson's distinction on these grounds and offer further considerations to avoid disability discrimination in emergency and crisis standards of care contexts.

\section{CONTEMPORARY RESEARCH ON THE CONCEPT OF DISABILITY}

Over the last thirty years, our understanding of the philosophy of disability has grown exponentially (Cureton and Wasserman 2020). Elizabeth Barnes' 2016 book The Minority Body marked a watershed moment by placing a decisive nail in the coffin of bad difference views of disability. Bad difference views hold that disability is by itself (a) something that makes one worse off; and (b) would still be "bad" even if society was fully accommodating of disabled people (Barnes 2016, 50). She instead defends a mere difference view of disability on which "having a disability is something that makes you different, but not something that by itself makes you worse off because of that difference" (Idem, 78, our italics). This does not mean that certain disabilities cannot engender negative consequences; on the contrary, that is certainly true. Her claim is instead about the prudential value of disability in general. Since the book's release, the field has considered and deliberated upon Barnes' arguments. Tellingly, the bad difference view of disability has continued to be broadly rejected, but there remains significant disagreement about the mere difference view. Barnes purposely limits her account to physical disabilities, and, unsurprisingly, most find it far less fitting as an account of many psychological disabilities. Others argue that conditions such as chronic pain challenge the mere-difference view on its own terms and are exceptions that should instead be understood as bad difference forms of disability (Campbell and Stramondo 2017).

\section{THE LIVED EXPERIENCES OF DISABILITY}

We will not take a side in this debate here. We have instead cursorily summarized the last five-plus years 